\title{
AN UNSUPERVISED PATCH-BASED APPROACH FOR EXOPLANET DETECTION BY DIRECT IMAGING
}

\section{Olivier Flasseur, Loïc Denis}

\author{
Laboratoire Hubert Curien UMR5516 \\ Univ Lyon, UJM-Saint-Etienne, CNRS, \\ Institut d Optique Graduate School \\ F-42023, Saint-Etienne, France
}

\begin{abstract}
The search for exoplanet is a very active topic in astronomy. Exoplanet detection by direct imaging requires both dedicated instruments to mask out the host star and careful image processing methods. Data processing is challenging because the exoplanet signal is very faint and hidden in a much stronger non-stationary background displaying strong spatial correlations. In contrast to previous detection methods, we explicitly model the spatial correlations of the background and design a completely unsupervised method that accounts for the background non-stationarity. From a time series of observations, we learn a local model of the distribution of background patches. Significant sources are then detected with a generalized likelihood ratio test. The sub-pixel location and flux of each detected exoplanet are estimated jointly to a refining of the background model. Each detected source is removed from the data, following an orthogonal matching pursuit strategy. The stopping criterion is based on a control of false alarms.

We compare the proposed algorithm to three state-of-theart exoplanet detection methods on datasets obtained with SPHERE instrument operating at the Very Large Telescope (VLT) in Chile. We show a drastic improvement of the sensibility and much fewer false detections.
\end{abstract}

Index Terms - Detection, estimation, shrinkage estimator, patch, covariance

\section{INTRODUCTION}

Evidence of the presence of exoplanets can either be indirect or direct. Examples of indirect detection techniques [1] include the analysis of the radial velocity of the host star [2], by Doppler effect, the planet's gravity resulting in the host star moving in a small orbit, or the transit photometry [3] which identifies the small drops of the star brightness when an exoplanets transits between the observer and the star. Direct observation of exoplanets $[4,5]$ is very challenging because of the small angular separation (a few arcseconds) and the huge brightness difference (typically in the range $10^{5}$ to $10^{7}$ in the near infrared) between the star and the exoplanets.

\author{
Éric Thiébaut, Maud Langlois
}

\author{
Centre de Recherche Astrophysique de Lyon \\ UMR5574 Université de Lyon, Université Lyon 1, \\ ENS de Lyon, CNRS \\ F-69230, Saint-Genis-Laval, France
}

Exoplanet detection by direct imaging is made possible by coupling high angular resolution (large telescopes with adaptive optics to compensate for atmospheric turbulence), partially masking of the star by a coronagraph [6], and differential imaging [7]. Differential imaging is based on the combination of several images in which the residual signal from the star and the signal of the exoplanets undergo different transforms. In angular differential imaging (ADI), the speckles observed when masking out the star are quasi-static while all off-axis sources (such as exoplanets) have an apparent rotation motion from one image to the other. Differential imaging and the detection technique form a crucial step that define the overall performance of the instrument.

Most of state-of-the-art methods for exoplanet detection in ADI perform first a subtraction step with the aim to suppress at best the speckles due to the star and hence improve the signal-to-noise ratio of the exoplanets. To process the image $r_{\ell}$ at time frame $t_{\ell}$, TLOCI algorithm and its variants $[8,9]$ estimate the linear combination of images $r_{m}$ recorded at times $t_{m} \neq t_{\ell}$ that reduces as much as possible the energy of the residuals $r_{\ell}-\sum_{m \neq \ell} \beta_{m} \cdot r_{m}$, with $\beta_{m}$ the weights of the linear combination. This subtraction can be improved by adjusting the weights $\beta_{m}$ over angular sectors [10]. An issue is the signal self-subtraction: because of the apparent motion of the field of view, the signal from the exoplanets is not located at the exact same position at other time frames $t_{m}$. However, that motion is small, especially at short angular separations, and part of the signal of interest is lost by this subtraction step. KLIP algorithm [11] improves the subtraction by computing the most significant modes with a principal components analysis (PCA). The observed images $\left\{r_{\ell}\right\}_{\ell=1: T}$ are then projected onto the subspace orthogonal to these modes, where the signal of the exoplanets remains mostly unchanged and the strong background due to the star is largely reduced. To improve the background subtraction, more modes must be included, at the cost of signal self-substraction. LLSG $[12,13]$ solves the signal self-substraction problem by decomposing the temporal stack of observations $\left\{r_{\ell}\right\}_{\ell=1: T}$ into a low-rank component (that mostly contains the background), 
a sparse component (that contains exoplanet signal) and a Gaussian noise component (that accounts for measurement noise). Unfortunately, it is difficult to control the false alarm rate when performing the detection on the sparse component due to the non-linear decomposition method used. The ANDROMEDA algorithm [14] applies a statistical detection approach to pre-filtered images where the background is approximately whitened by computing both time differences and a band-pass spatial filtering.

Existing methods require a trained user to hand-tune several parameters. They compensate for the lack of stationarity in the detection maps by some ad-hoc normalization steps and correct the loss of signal due to signal self-subtraction by a calibration step where fake planets are first injected in the data, then detected to locally estimate the bias.

Our contributions: We propose a new and entirely unsupervised method named PACO (for PAtch COvariance) which is statistically grounded and photometrically unbiased for the detection of exoplanets in direct imaging. Rather than subtracting a global model of the background, we locally learn a statistical model of background fluctuations, at the scale of a patch, and capture the spatial correlations of the background. Our detection is based on a generalized likelihood ratio test. We show on real data that false alarms are controlled, so that a single threshold can be set to decide for statistically relevant detections. The same statistical model of the background can be used to estimate the flux (i.e. the photometry) of the detected exoplanets.

Our method is described in section 2. We show in section 3 that the detection performance is significantly improved compared to state-of-the-art methods.

\section{EXOPLANET DETECTION BY MODELING BACKGROUND PATCHES COVARIANCE}

Fig. 1 illustrates an ADI dataset: $T$ images in which the dominant component corresponds to speckles due to the star masked by the coronagraph. In these images, exoplanets are too faint to be visible. We propose to learn a local model of the background from the collection of patches extracted at each location of the field of view. Patch-based models have been widely used in image processing, Gaussian models have in particular led to very efficient denoising techniques $[15,16]$.

Statistical model of the background: Due to the motion of the field of view from one time to another, an exoplanet initially located at angular position $\phi_{0}$ on a reference frame is located at $\phi_{t}=\mathcal{F}_{t}\left(\phi_{0}\right)$ at time $t$, see Fig. 1. Exoplanets are very faint and very few so that it is unlikely that the signal from two exoplanets overlap. We model the recorded intensity $r_{\theta_{k}, t_{\ell}}$ at the 2-D pixel location $\theta_{k}$ and time $t_{\ell}$ by the superimposition an unresolved exoplanet and the background component:

$$
r_{\theta_{k}, t_{\ell}}=\alpha h_{\theta_{k}}\left(\phi_{t_{\ell}}\right)+f_{\theta_{k}, t_{\ell}}
$$

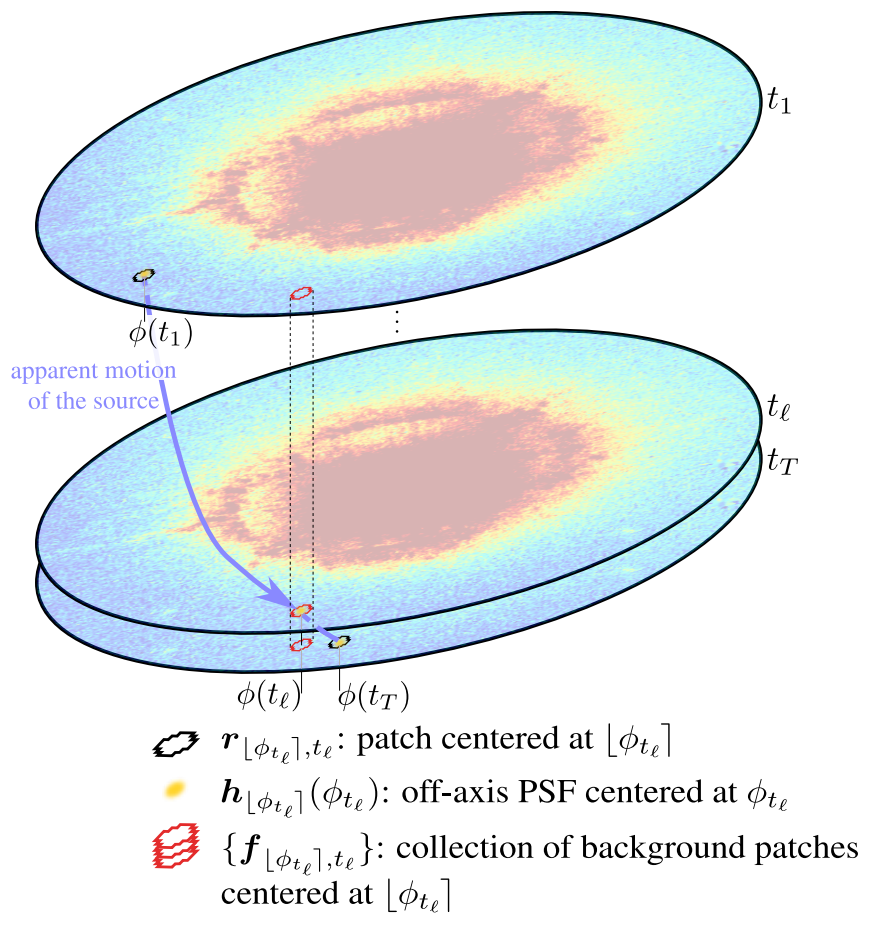

Fig. 1. An ADI dataset consists of $T N$-pixels images in which the dominant signal (the central red region) corresponds to speckles due to the star, and the signal of interest is due to the faint sources that undergo a (known) rotation from one time to another. Our method locally models the background by analyzing the collection of patches centered at a given location of the field of view.

with $\alpha \geq 0$ the flux of the exoplanet, $h_{\theta_{k}}\left(\phi_{t_{\ell}}\right)=h\left(\theta_{k}-\phi_{t_{\ell}}\right)$ the off-axis PSF, centered on the location $\phi_{t_{\ell}}$ of the exoplanet at time $t_{\ell}$ and sampled at pixel location $\theta_{k}$, and $f_{\theta_{k}, t_{\ell}}$ the background at spatio-temporal index $(k, \ell)$. Under a statistical model of the background specifying the probability density function $\mathrm{p}_{f}$, the maximum likelihood estimator of the flux $\alpha$ of a potential source located at position $\phi_{0}$ in the reference frame is:

$$
\widehat{\alpha}=\underset{\alpha}{\arg \max } \mathrm{p}_{f}\left(\left\{r_{\theta_{k}, t_{\ell}}-\alpha h_{\theta_{k}}\left(\mathcal{F}_{t_{\ell}}\left(\phi_{0}\right)\right)\right\}_{k=1: N, \ell=1: T}\right),
$$

and the detection can be expressed by the binary hypothesis test:

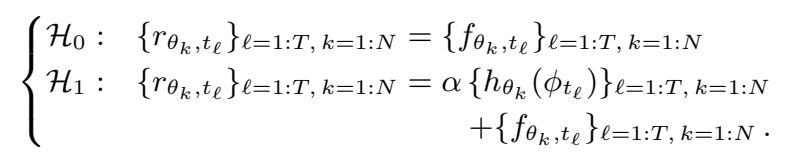

Under hypothesis $\mathcal{H}_{0}$, no exoplanet is present at location $\phi_{0}$ ( $\alpha=0$ in eq. (1)). The detection can be performed via the generalized likelihood ratio test:

$$
\log \frac{\mathrm{p}_{f}\left(\left\{r_{\theta_{k}, t_{\ell}}-\widehat{\alpha} h_{\theta_{k}}\left(\phi_{t_{\ell}}\right)\right\}_{k=1: N, \ell=1: T}\right)}{\mathrm{p}_{f}\left(\left\{r_{\theta_{k}, t_{\ell}}\right\}_{k=1: N, \ell=1: T}\right)} \underset{\mathcal{H}_{0}}{\stackrel{\mathcal{H}_{1}}{\gtrless}} \eta .
$$

In contrast to other exoplanet detection methods, we do not consider background speckles as a deterministic component 
but as a random fluctuation. Given the non-stationarity of the background in the field of view, we build a local model, based on patches. In the following, bold face letters indexed by a location indicate $K$-pixels disk-shaped 2D patches extracted at that spatial location. We model the distribution of the collection of background patches $\left\{\boldsymbol{f}_{\theta_{k}, t_{\ell}}\right\}_{\ell=1: T}$ extracted at some location $\theta_{k}$ (red patches in Fig. 1) by a multivariate Gaussian $\mathcal{N}\left(\boldsymbol{m}_{\theta_{k}}, \mathbf{C}_{\theta_{k}}\right)$ with a full covariance (hence the name of our method: PACO, for PAtch COvariance).

We estimate the mean $\boldsymbol{m}_{\theta_{k}}$ using the sample mean. Due to the limited number $T$ of temporal frames, estimation of $\mathbf{C}_{\theta_{k}}$ with the sample covariance estimator leads to rank deficient matrices. It is thus necessary to regularize the estimation. We use the shrinkage estimator for covariance matrices proposed by [17] and defined by: $\widehat{\mathbf{C}}=(1-\widehat{\rho}) \widehat{\mathbf{S}}+\widehat{\rho} \widehat{\mathbf{F}}$, where $\widehat{\mathbf{S}}$ is the sample covariance matrix (unbiased but suffering from a large variance) and $\widehat{\mathbf{F}}$ is the diagonal matrix formed from the sample variances (biased but with a limited variance). Parameter $\hat{\rho}$ balances each estimator to reach a bias-variance tradeoff. By extending the results of [17] to the specific form of $\widehat{\mathbf{F}}$, we get the data-driven expression:

$$
\hat{\rho}\left(\widehat{\mathbf{S}}_{\theta_{k}}\right)=\frac{\operatorname{tr}\left(\widehat{\mathbf{S}}_{\theta_{k}}^{2}\right)+\operatorname{tr}^{2}\left(\widehat{\mathbf{S}}_{\theta_{k}}\right)-2 \sum_{i=1}^{K}\left[\widehat{\mathbf{S}}_{\theta_{k}}\right]_{i i}^{2}}{(T+1)\left(\operatorname{tr}\left(\widehat{\mathbf{S}}_{\theta_{k}}^{2}\right)-\sum_{i=1}^{K}\left[\widehat{\mathbf{S}}_{\theta_{k}}\right]_{i i}^{2}\right)}
$$

Estimation of the exoplanet flux: With our multivariate Gaussian model of the background, the maximum likelihood estimator (2) of the exoplanet flux is $\widehat{\alpha}=b / a$, with

$$
\left\{\begin{array}{l}
a=\sum_{\ell=1}^{T} \boldsymbol{h}_{\left\lfloor\phi_{t_{\ell}}\right\rceil}\left(\phi_{t_{\ell}}\right)^{\mathrm{t}} \cdot \widehat{\mathbf{C}}_{\left\lfloor\phi_{\left.t_{\ell}\right\rceil}\right.}^{-1} \cdot \boldsymbol{h}_{\left\lfloor\phi_{\left.t_{\ell}\right\rceil}\right.}\left(\phi_{t_{\ell}}\right) \\
b=\sum_{\ell=1}^{T} \boldsymbol{h}_{\left\lfloor\phi_{\left.\ell_{\ell}\right\rceil}\right.}\left(\phi_{t_{\ell}}\right)^{\mathrm{t}} \cdot \widehat{\mathbf{C}}_{\left\lfloor\phi_{\left.t_{\ell}\right\rceil}\right\rceil}^{-1} \cdot\left(\boldsymbol{r}_{\left\lfloor\phi_{\left.t_{\ell}\right\rceil}\right\rceil t_{\ell}}-\widehat{\boldsymbol{m}}_{\left\lfloor\phi_{\left.t_{\ell}\right\rceil}\right.}\right),
\end{array}\right.
$$

where $\boldsymbol{h}_{\left\lfloor\phi_{\left.t_{\ell}\right\rceil}\right.}\left(\phi_{t_{\ell}}\right)$ stands for the off-axis PSF for a source at sub-pixelic location $\phi_{t_{\ell}}$ sampled over a patch whose center is $\left\lfloor\phi_{t_{\ell}}\right\rceil$, the nearest pixel to $\phi_{t_{\ell}}$. The standard-deviation of $\widehat{\alpha}$ is $\widehat{\sigma}_{\alpha}=1 / \sqrt{a}$. Given that the flux of an exoplanet is necessarily positive, we define $\widehat{\alpha}^{+}=\max (\widehat{\alpha}, 0)$.

Detection of an exoplanet: The generalized likelihood ratio test defined in (4), under positivity constraint, is given by:

$$
\left(\mathrm{GLRT}^{+}\right) \frac{\max (b, 0)^{2}}{a} \underset{\mathcal{H}_{0}}{\stackrel{\mathcal{H}_{1}}{\gtrless}} \eta \text {. }
$$

When $\eta \geq 0$, this test is equivalent to the test $\widehat{\alpha} / \widehat{\sigma}_{\alpha} \gtrless \tau$ with $\tau=\sqrt{\eta}$. This latter test corresponds to a linear transform of the data and can be interpreted as the signal-to-noise ratio (SNR) of the estimation of the (unconstrained) flux $\alpha$ of the source. It is straightforward to show that, under our Gaussian model, it follows a standard normal distribution. Under hypothesis $\mathcal{H}_{0}$, the threshold value $\tau$ can thus easily be translated into a false alarms probability.

Once an exoplanet has been detected (the test being larger than the threshold $\tau$ at some location $\phi_{0}$ ), the flux $\alpha$ can be improved by jointly estimating $\alpha$ and the background statistics (mean and covariances of the Gaussian models at each

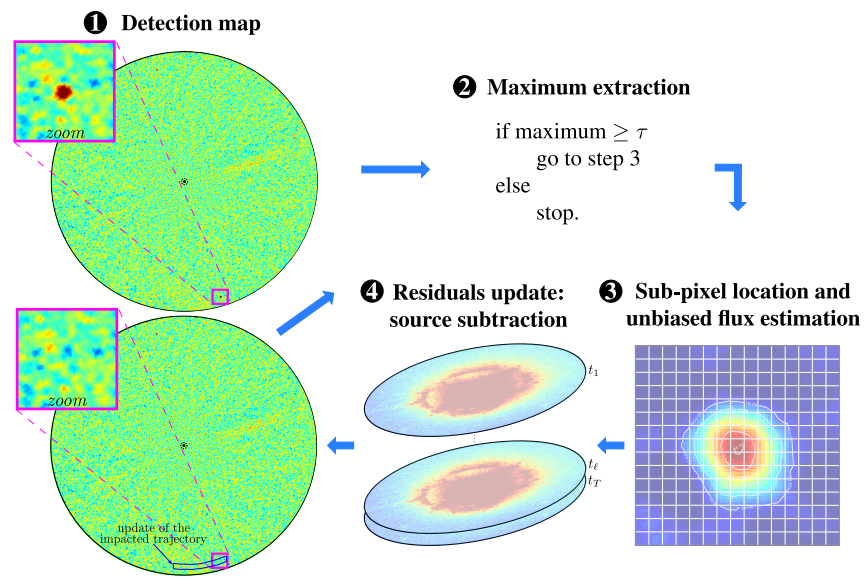

Fig. 2. Synopsis of PACO algorithm.

location $\left\{\phi_{t_{\ell}}\right\}_{\ell=1: T}$ at which the planet is seen at the corresponding times $t_{1}$ to $t_{T}$ ).

PACO, our exoplanet detection algorithm: Figure 2 summarizes our exoplanet detection algorithm. In step (1, the local patch mean and patch covariances are computed. Then, a detection map is obtained by computing the SNR $\widehat{\alpha} / \widehat{\sigma}_{\alpha}$ at each possible locations $\phi_{0}$ within the field of view. Step 2 checks whether the largest value in the detection map is above the detection threshold. If not, the algorithm stops, otherwise, an exoplanet is detected at the location of the maximum of the detection map. Step 3 then refines the location to sub-pixelic accuracy and improves the estimation of the flux $\alpha$ of the detected exoplanet by jointly estimating $\alpha$ and the background means and covariances. This joint estimation procedure ${ }^{1}$ prevents from any signal self-subtraction (removal of part of the exoplanet signal in the background mean). Once this local optimization step is finished (the convergence criterion is based on the computed Cramér-Rao lower bounds on localization and flux accuracies), step 4 removes the signal of the detected exoplanet from the data. Step 1 is then repeated on the residual signal, and the subsequent steps, until the condition in step 2 indicates that no more statistically significant detections can be made.

The method is unsupervised: no parameter tuning is necessary, the patch size is set based on a Monte Carlo study and is constant for a given instrument ${ }^{2}$; the threshold $\tau$ is set according to a prescribed false alarms rate ${ }^{3}$.

\footnotetext{
${ }^{1}$ if two overlapping sources are detected, their flux is jointly estimated like in a conventional orthogonal matching pursuit procedure

2 it is related to the diffraction limit of the telescope, and we found that a disk-shaped patch with 49 pixels was optimal for SPHERE-IRDIS instrument

${ }^{3}$ it is common practice to set $\tau=5$ for exoplanet detection, leading to a probability of false alarm below $2.9 \times 10^{-7}$
} 

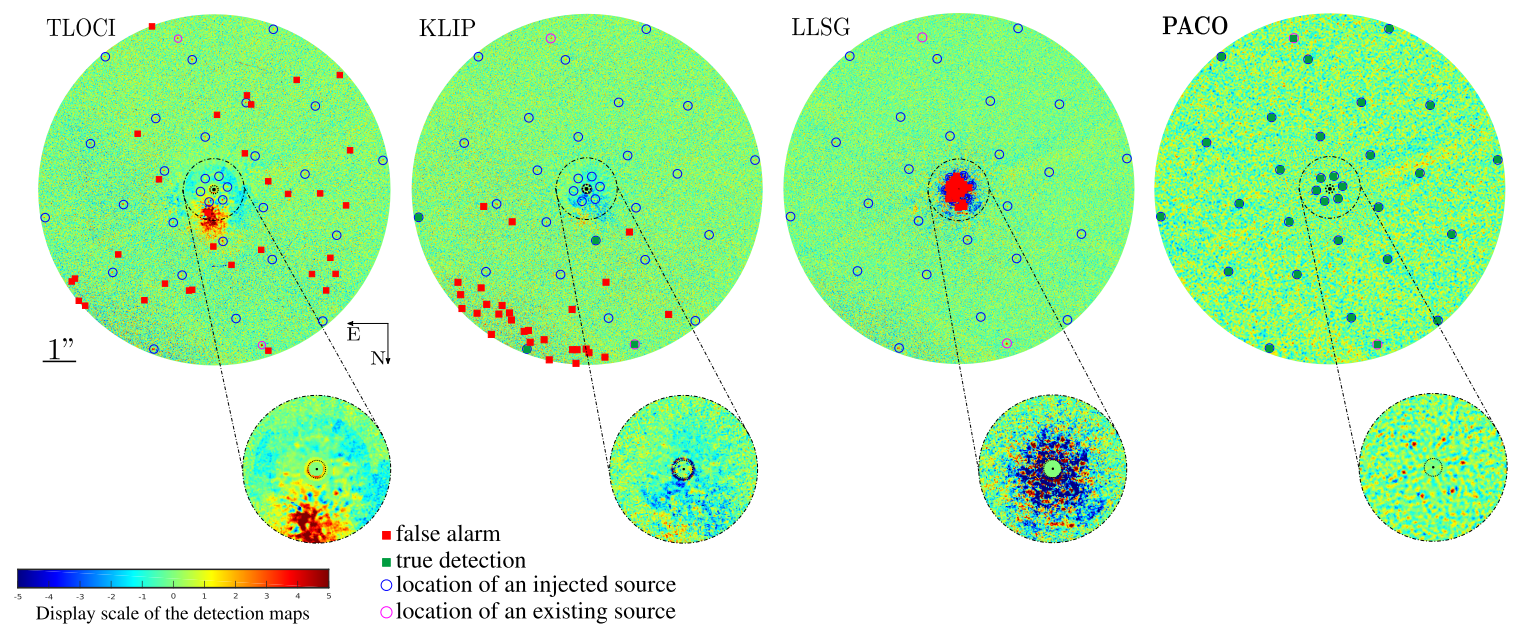

Fig. 3. Detection maps obtained with PACO, TLOCI, KLIP and LLSG algorithms. The first 32 detections are marked on each map by square patterns.

\section{PERFORMANCE OF THE PROPOSED METHOD}

Detection maps obtained with PACO algorithm are compared to the maps produced by TLOCI [9], KLIP [11] and LLSG [12] algorithms presented in the introduction. TLOCI and KLIP maps are obtained with the VLT/SPHERE reduction pipeline (SpeCal) [18] and thresholded, as done routinely for data analysis. We used data obtained by the InfraRed Dual Imaging Spectrograph (IRDIS) of the Spectro-Polarimetry High-contrast Exoplanet REsearch (SPHERE) instrument operating on the Very Large Telescope (VLT), in Chile. We processed a temporal stack of 96 frames acquired on the star HIP72192. There are two known faint point sources in the field of view ${ }^{4}$. We added 30 synthetic exoplanet signals, with faint brightness (mean flux $\alpha$ between $2.5 \times 10^{-6}$ and $3.9 \times 10^{-5}$ that of the host star, depending on the angular separation), in order to compare the capability of the different algorithms to recover them.

Figure 3 shows the detection maps computed with each method. Figure 4 gives the corresponding receiver operating characteristic (ROC) curves representing the true positive rate (TPR) as a function of the full-frame false detection number. Both figures illustrate the superior detection performance of PACO for faint sources (all sources are detected) and the improved behavior with respect to false alarms (no false alarm larger than 4 within the field of view). State-of-the-art algorithms are very sensitive to false alarms, particularly near the host star which drastically limit their detection ability in this area where the detection is the most difficult due to the small apparent rotation of the field of view. Our forthcoming paper [19] gives several additional results and shows that contrary to existing algorithms [20,21], the probability of false alarm is reliably controlled when thresholding the SNR maps.

\footnotetext{
${ }^{4}$ the question of whether these sources are actual exoplanets or brown dwarfs behaving as exoplanets is still open
}

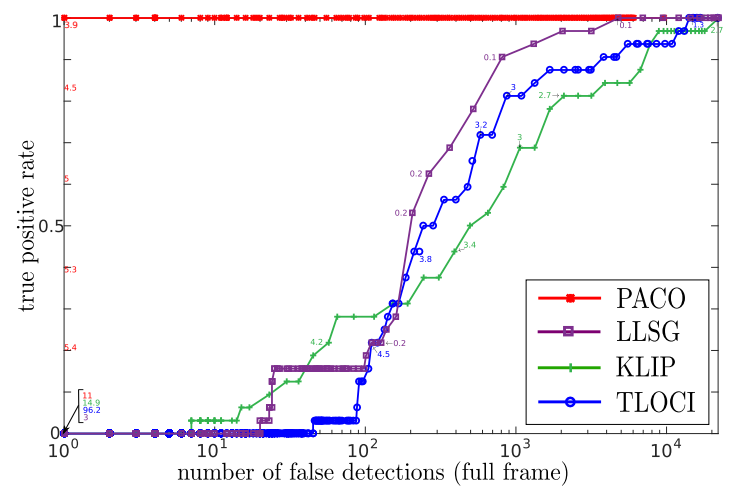

Fig. 4. ROC curves for each exoplanet detection method.

\section{CONCLUSION}

We proposed a new method (PACO) for exoplanet detection by direct imaging. Our method differs from existing approaches by its local modeling of the background fluctuations. We take advantage of the different backgrounds available in the temporal stack to learn a local multi-variate Gaussian. This model captures the background spatial correlations at a local scale of small patches. This improves the robustness to fluctuations of the background due to the stellar leakages or the evolution of the quality of the adaptive optics correction. We proposed a method to iteratively extract exoplanets until no detection is statistically relevant given the chosen false alarm rate. Results on real observations from VLT/SPHERE-IRDIS instrument show a clear advantage of our algorithm compared to existing methods based on image subtraction or image decomposition approaches, in particular at small angular separations, a case that is very important in the quest for exo-Earth. 


\section{REFERENCES}

[1] N.C. Santos, "Extra-solar planets: Detection methods and results," New Astronomy Reviews, vol. 52, no. 2, pp. 154-166, 2008.

[2] Y. Shen and E.L. Turner, "On the eccentricity distribution of exoplanets from radial velocity surveys," The Astrophysical Journal, vol. 685, no. 1, pp. 553, 2008.

[3] D. Charbonneau, T.M. Brown, A. Burrows, and G. Laughlin, "When extrasolar planets transit their parent stars," arXiv preprint astro-ph/0603376, 2006.

[4] W.A. Traub and B.R. Oppenheimer, "Direct imaging of exoplanets," Exoplanets, pp. 111-156, 2010.

[5] A. Vigan, C. Moutou, M. Langlois, F. Allard, et al., "Photometric characterization of exoplanets using angular and spectral differential imaging," Monthly Notices of the Royal Astronomical Society, vol. 407, no. 1, pp. 71-82, 2010.

[6] D. Mawet, P. Riaud, J. Baudrand, P. Baudoz, et al., "The four-quadrant phase-mask coronagraph: white light laboratory results with an achromatic device," Astronomy \& Astrophysics, vol. 448, no. 2, pp. 801-808, 2006.

[7] C. Marois, D. Lafreniere, R. Doyon, B. Macintosh, et al., "Angular differential imaging: A powerful highcontrast imaging technique," The Astrophysical Journal, vol. 641, no. 1 , pp. 556, 2006.

[8] C. Marois, C. Correia, J-P. Véran, and T. Currie, "Tloci: A fully loaded speckle killing machine," Proceedings of the International Astronomical Union, vol. 8, no. S299, pp. 48-49, 2013.

[9] C. Marois, C. Correia, R. Galicher, P. Ingraham, et al., "Gpi psf subtraction with tloci: the next evolution in exoplanet/disk high-contrast imaging," arXiv preprint arXiv:1407.2555, 2014

[10] Z. Wahhaj, L.A. Cieza, D. Mawet, B. Yang, et al., "Improving signal-to-noise in the direct imaging of exoplanets and circumstellar disks with mloci," Astronomy \& Astrophysics, vol. 581, pp. A24, 2015.

[11] R. Soummer, L. Pueyo, and J. Larkin, "Detection and characterization of exoplanets and disks using projections on karhunen-loève eigenimages," The Astrophysical Journal Letters, vol. 755, no. 2, pp. L28, 2012.

[12] C.A. Gomez Gonzalez, O. Absil, P.A. Absil, M. Van Droogenbroeck, et al., "Low-rank plus sparse decomposition for exoplanet detection in directimaging adi sequences-the llsg algorithm," Astronomy \& Astrophysics, vol. 589, pp. A54, 2016.
[13] C.A. Gomez Gonzalez, O. Wertz, O. Absil, V. Christiaens, et al., "Vip: Vortex image processing package for high-contrast direct imaging," The Astronomical Journal, vol. 154, no. 7, pp. 12pp, 2017.

[14] F. Cantalloube, D. Mouillet, L.M. Mugnier, J. Milli, et al., "Direct exoplanet detection and characterization using the andromeda method: Performance on vlt/naco data," Astronomy \& Astrophysics, vol. 582, pp. A89, 2015.

[15] D. Zoran and Y. Weiss, "From learning models of natural image patches to whole image restoration," in Computer Vision (ICCV), 2011 IEEE International Conference. IEEE, 2011, pp. 479-486.

[16] M. Lebrun, A. Buades, and J.M. Morel, "Implementation of the non-local bayes (nl-bayes) image denoising algorithm," Image Processing On Line, vol. 2013, pp. $1-42,2013$.

[17] Y. Chen, A. Wiesel, Y.C. Eldar, and A.O. Hero, "Shrinkage algorithms for mmse covariance estimation," IEEE Transactions on Signal Processing, vol. 58, no. 10, pp. 5016-5029, 2010.

[18] R. Galicher, A. Boccaletti, D. Mesa, P. Delorme, et al., "Astrometric and photometric accuracies in high contrast imaging: The sphere speckle calibration tool (specal)," accepted in Astronomy \& Astrophysics, 05/2018.

[19] O. Flasseur, L. Denis, É. Thiébaut, and M. Langlois, "Exoplanet detection in angular differential imaging by statistical learning of the nonstationary patch covariances," accepted in Astronomy \& Astrophysics, 03/2018.

[20] C. Marois, D. Lafrenière, B. Macintosh, and R. Doyon, "Confidence level and sensitivity limits in high-contrast imaging," The Astrophysical Journal, vol. 673, no. 1, pp. 647-656, 2008.

[21] D. Mawet, J. Milli, Z. Wahhaj, and D. Pelat, "Fundamental limitations of high contrast imaging set by small sample statistics," The Astrophysical Journal, vol. 792, no. 2, pp. 97-107, 2014. 